\title{
Single-Cell Chromatin Accessibility Landscape of Human Umbilical Cord Blood in Trisomy 18 Syndrome
}

\section{Xiaofen Qiu}

Shenzhen People's Hospital

Haiyan Yu

Shenzhen People's Hospital

Hongwei Wu

Shenzhen People's Hospital

Zhiyang Hu

Shenzhen People's Hospital

Jun Zhou

Shenzhen People's Hospital

\section{Hua Lin}

People's Liberation Army Joint Logistic Support Force 924th Hospital: 181st Hospital of Chinese People's Liberation Army

\section{Wen Xue}

People's Liberation Army Joint Logistic Support Force 924th Hospital: 181st Hospital of Chinese People's Liberation Army

\section{Wanxia Cai}

Shenzhen People's Hospital

\section{Jiejing Chen}

People's Liberation Army Joint Logistic Support Force 924th Hospital: 181st Hospital of Chinese People's Liberation Army

\section{Qiang Yan}

People's Liberation Army Joint Logistic Support Force 924th Hospital: 181st Hospital of Chinese People's Liberation Army

\section{Weier Dai}

University of Texas at Austin Architecture and Planning Library: The University of Texas at Austin

\section{Ming Yang}

People's Liberation Army Joint Logistic Support Force 924th Hospital: 181st Hospital of Chinese

People's Liberation Army

\section{Donge Tang}

Shenzhen People's Hospital 


\section{Yong Dai ( $\nabla$ daiyong22@aliyun.com )}

Shenzhen People's Hospital https://orcid.org/0000-0002-6840-9158

\section{Research Article}

Keywords: Trisomy 18 Syndrome, single-cell sequencing, Transcription factors, aneuploidy, Developmental regulation

Posted Date: April 26th, 2021

DOl: https://doi.org/10.21203/rs.3.rs-327935/v1

License: (c) (1) This work is licensed under a Creative Commons Attribution 4.0 International License. Read Full License 


\section{Abstract}

Background: Trisomy 18 syndrome (Edwards syndrome, ES) is a type of aneuploidy caused by the presence of an extra chromosome 18. Aneuploidy is the leading cause of early pregnancy loss, intellectual disability, and multiple congenital anomalies.

Results: In this study, we used the commercial Chromium platform (10x Genomics) to perform sc-ATACseq to measure chromatin accessibility in 11,611 single umbilical cord blood cells derived from one trisomy 18 syndrome patient and one healthy donor. We obtained 13 distinct major clusters of cells and identified them as 6 human umbilical cord blood mononuclear cell types without using antibodies. We set out to generate a single-cell atlas of chromatin accessibility in human umbilical cord blood from the healthy control donor and the 18 trisomy syndrome donor. Then, we carried out cell-type-specific gene regulatory network analysis at single-cell resolution of these differential accessibility-related loci genes and summarized the disease-related transcription factors (TFs) and target genes. Finally, we performed a cell-type-specific gene regulatory network analysis of these differential accessibility-related locus genes at single-cell resolution.

Conclusions: Specifically, CCBN2 and MCM3 may be essential for the development of trisomy 18, and genes differentially expressed between the donor and patient were enriched in the human T-cell leukaemia virus 1 infection pathway. These screened disease-related transcription factors (TFs) and their target genes provide a basis for further research that will improve our understanding of trisomy 18 syndrome.

\section{Introduction}

Trisomy 18 syndrome is a type of common aneuploidy caused by the presence of an extra full chromosome 18 trisomy, mosaic trisomy, or partial trisomy 18(Cereda and Carey, 2012). Edwards et al described the first reported infants in 1960, it is also known as Edwards Syndrome (ES)(Edwards et al., 1960;Smith et al., 1960). ES is the second most common autosomal trisomy syndrome with a frequency of around 1 in 6000 live births after trisomy 21 (known as down syndrome)(Goel et al., 2019). Patients with trisomy 21 can survive as chromosome 21 is the minimal number of chromosomes transcribed in humans, but Trisomy 18 is viable after birth but die within a few months of birth (Torres et al., 2008). The main clinical features include prenatal growth deficiency, characteristic craniofacial features, craniofacial features, distinctive hand posture of overriding fingers, nail hypoplasia, short hallux, and major malformations (particularly involving the heart)(Carey, 2005). It is generally believed that the extra chromosome exists because of non-disjunction. In parental origin analysis, additional chromosomes are usually of maternal origin as a result of chromosomal segregation erroneous in meiosis or post-zygotic mitosis(Fisher et al., 1995;Kupke and Müller, 1989). A study has shown changes in gene expression in trisomy 18 syndrome. The British team of David R. Fitz Patrick, based on cDNA array analysis of trisomy syndromes, found that three-body on chromosome gene transcription, on average, increased only 1.1 times. They detected the expression level differences between chromosomes, suggesting that the 
genomic regulation mechanisms may act at greater distances than ever thought; that is, a chromosome increase may affect other chromosomes gene transcription levels. Their data support a model, in which trisomy leads to slightly raised on chromosome gene leads to secondary transcription, universality, and more extreme imbalance, and the extent of this faulty regulation may determine the severity of the phenotype(FitzPatrick et al., 2002). To date, we know little about the genomic regulatory of trisomy 18 syndrome. Human aneuploidy is the major cause of early pregnancy loss, intellectual disability, and multiple congenital anomalies, and trisomy is the most common aneuploidy in humans(R et al., 2013). Previous studies focused mostly on Trisomy 21 syndrome, however, the pathogenic mechanisms of trisomy 18 syndrome remain largely unknown due to the high mortality associated with aneuploidy(Zhang et al., 2013). There is an urgent need to understand the mechanisms of gene expression regulation in trisomy 18.

Traditional high-throughput sequencing strategies that use intact tissue or cells are limited to averaging the constituent cell profiles. Thanks to great advancements in unbiased single-cell genomic assays, a single-cell assay for transposase-accessible chromatin using sequencing (sc-ATAC-seq) has been developed(Satpathy et al., 2019). It leverages the concept that every single cell can only occupy a single position in the landscape of cell types. This assay can reveal several layers of gene regulation in a single assay, including genome-wide identification of cis-elements, inference with TF binding and activity, and nucleosome positions (Buenrostro et al., 2013;Schep et al., 2015). Sc-ATAC-seq not merely adapted to disentangling cell type heterogeneity, but it also to captures the chromatin regulatory landscape that governs transcription in each cell type in complex tissues. To characterize the cellular and molecular changes associated with trisomy 18 syndrome in greater detail, and to gain further insights into the mechanisms influencing one trisomy 18 syndrome traits, we subjected human umbilical cord blood of the trisomy 18 syndrome, patient and one control to asingle-cell assay for transposase accessible chromatin using sequencing (sc-ATAC-seq). The latest-generation DNA sequencing technology, single-cell assay for transposase-accessible chromatin (sc-ATAC-seq) is expected to eventually reveal the mechanisms of gene expression regulation and the related specific molecular markers in cell clusters.

In this approach, we applied a commercial system (10x Genomics) to perform sc-ATAC-seq on nanolitescale droplets, which can generate high-quality single-cell chromatin accessibility profiles on a large scale. We performed sc-ATAC-seq to measure chromatin accessibility in 11,611 single cells derived from 2 samples, one trisomy 18 syndrome patient and one healthy donor. Using the sc-ATAC-seq data, we obtained 11 distinct major clusters of cells, identified as 6 human umbilical cord blood mononuclear cell types antibody free, and characterized the driving regulatory factors and differentially accessible loci that defined each cluster. We set out to generate a single-cell atlas of chromatin accessibility from healthy and the trisomy syndrome human umbilical cord blood samples. Finally, cell-type-specific gene regulatory network analysis of these differential accessibility-related loci genes was carried out at single-cell resolution, and disease-related genes were predicted. These screened disease-related transcription factors (TFs) and their target genes provide a basis for further research that will improve our understanding of trisomy 18 syndrome. 


\section{Method}

\subsection{Study group}

The criteria for inclusion in the disease group for this study were $\mathrm{G}$ banding karyotype was $47, \mathrm{XY},+18$ according to the international system for human cytogenetic nomenclature(ISCN), 2016. One umbilical cord blood (UCB) sample was collected with informed consent from a 32-year-old, pregnant, healthy donor as a natural control (NC). The G banding karyotype of the healthy control woman's foetus was 46 , $X Y$. Patients UCB samples recruited for this study were collected with informed consent from a 20-yearold pregnant donor as the study subject (trisomy 18 syndrome, also known as Edwards syndrome, ES). The $\mathrm{G}$ banding karyotype of this donor's foetus was $47, \mathrm{XY},+18$. Each foetus was at a gestational age of 18-22 weeks. Samples were stored in ethylene diamine tetraacetic acid (EDTA) anticoagulant tubes and transported to the laboratory within 1 hour. Cord blood mononuclear cells (CBMCs) were isolated by Ficoll-Hypaque density gradient centrifugation according to standard protocols.

\subsection{Sc-ATAC-seq using the 10x Chromium platform.}

All protocols of this study to generate scATAC-seq data on the 10x Chromium platform, including nucleus isolation \& transposition, library construction, and instrument and sequencing parameters, are described below and available here: https://support.10xgenomics.com/single-cell-atac.

\subsubsection{Nuclei isolation \& Transposition}

The CBMC thawing, isolation, washing, and counting of nuclear suspensions were performed according to the Demonstrated Protocol: Nuclei Isolation for Single Cell ATAC Sequencing (10x Genomics). Step 1: CBMCs were gently thawed in a water bath at $37^{\circ} \mathrm{C}$ for $1-2$ min and resuspended in PBS $+0.04 \% \mathrm{BSA}$. Step 2: 100,000-1,000,000 cells were added to a 2-ml microcentrifuge tube and centrifuged at $300 \mathrm{rcf}$ for $5 \mathrm{~min}$ at $4^{\circ} \mathrm{C}$. All supernatant was removed without disrupting the cell pellet. Step 3: add $100 \mu$ of chilled lysis buffer(10 mM Tris-HCl (pH 7.4), 10 mM NaCl, 3 mM MgCl2, 0.1\% Tween-20, 0.1\% Nonidet P40 Substitute, $0.01 \%$ digitonin and $1 \%$ BSA) was added. The sample was incubated for 3 min on ice. Step 4: add $1 \mathrm{ml}$ chilled wash buffer (10 mM Tris- $\mathrm{HCl}(\mathrm{pH} 7.4), 10 \mathrm{mM} \mathrm{NaCl}, 3 \mathrm{mM} \mathrm{MgCl} 2,0.1 \%$ Tween-20 and $1 \%$ BSA) was added to the lysed cells, which were mixed with a pipette $5 x$ and centrifuged at 500 rcf for 5 $\min$ at $4^{\circ} \mathrm{C}$. The supernatant was removed without disrupting the nuclear pellet. Based on the nuclear concentration mesured before step 2 and assuming $~ 50 \%$ nuclear loss during cell lysis, the nuclear pellet was resuspended in chilled diluted Nuclei Buffer (10x Genomics, PN-2000153). The cell suspension was was passed through a $40 \mu \mathrm{m}$ Flowmi Cell Strainer, and the cell concentration was determined using a Countess II FL Automated Cell Counter or a heamocytometer. We proceeded immediately to the Chromium Single Cell ATAC Reagent Kits protocol (CG000168). Nuclear suspensions were incubated in a transposition mix that included a transposase. The transposase entered the nuclei and preferentially 
fragmented the DNA in regions of the accessible chromatin while adapter sequences were added to the ends of the DNA fragments.

\subsubsection{Sc-ATAC-seq library construction}

Sc-ATAC-seq libraries were prepared according to the Chromium Single Cell ATAC Reagent Kits User Guide (10x Genomics; CG000168 Rev B). Briefly, nuclei were transposed in a bulk solution; then, using a microfluidic chip, the nuclei were partitioned into nanolitre-scale gel beads-in-emulsion (GEMs). GemCode Technology sampled a pool of $\sim 750,00010 x$ barcodes to separately and uniquely index the transposed DNA of each individual cell. GEMs were generated by combining barcoded gel beads, transposed nuclei, a master mix, and partitioning oil on a Chromium Chip E. The nuclei were delivered at limiting dilution to achieve single-nucleus resolution; therefore, the majority ( 90-99\%) of generated GEMs contained no nuclei, while the remainder largely contained a single nucleus. The gel bead was dissolved after GEM generation. Oligonucleotides containing an Illumina ${ }^{\circledR}$ P5 sequence, a 10x barcode and a Read 1 (Read $1 \mathrm{~N})$ sequence were released and mixed with DNA fragments and master mix. The thermal cycling of the GEMs produced 10x-barcoded single-stranded DNA. After incubation, the GEMs were broken, and pooled fractions were recovered. Next, silane magnetic beads were used to remove leftover biochemical reagents from the post-GEM reaction mixture. Solid-phase reversible immobilization (SPRI) beads were used to eliminate unused barcodes from the sample. In the end, the sample index P7 and Read 2 (Read 2N) sequences were added during library construction via PCR. The final ScATAC-seq libraries contained the P5 and P7 primers used in Illumina ${ }^{\circledR}$ bridge amplification. Libraries were generated and sequenced, and $10 \mathrm{x}$ barcodes were used to associate individual reads with the individual partitions and thereby to each cell.

\subsubsection{Data Quality Control and Genome Alignment}

To improving data quality, fix the occasional sequencing error in barcodes, the fragments get associated with the original barcodes. The barcode sequence is first obtained from the "I2" index reads. Cell Ranger ATAC(https://support.10xgenomics.com/single-cell-atac/software/overview/welcome) performs reference-based analysis and requires adapter and primer oligo sequence to be trimmed off before mapping confidently. Use the cut adapt(Criscuolo and Brisse, 2013) tool to identify the primer sequence at the end of each read and trim it from the read before alignment. Then, the trimmed read-pairs are aligned to a specified reference using BWA-MEM(Clevenger et al., 2015) with GRCh38. Reads less than 25 bp are not aligned by BWA-MEM. These unaligned reads are marked as unmapped. Due to PCR amplification, a barcode fragment is sequenced multiple times. Duplicate reads are obtained by identifying a set of read pairs that have the same mapping position on the reference genome at the $5^{\prime}$ end of all barcodes, where R1 and R2 have the same mapping position on the reference genome. Unique read pairs were reported as a fragment in the fragment file. 


\subsubsection{Dimensionality Reduction \& Clustering and UMAP Projection}

We used Signac v0.2.5 (https://github.com/timoast/signac) and Seurat(Stuart et al., 2019) performs clustering and UMAP projection. Since the data under the single-cell resolution is sparse, we first perform dimensionality reduction to cast it into a lower-dimensional space, which has the advantage of denoising. At the same time, Cell Ranger ATAC supports dimensionality reduction via Latent Semantic Analysis (LSA)(González et al., 2016). We performed a graph-based clustering and visualization via UMAP(Kobak and Berens, 2019). The data normalized to the unit norm before performing graph-based clustering and UMAP projection.

\subsubsection{Marker gene identification and cell specific-type annotation}

Marker gene identification and cell specific-type annotation were performed according to the Cell Type Annotation Strategies for Single Cell ATAC-Seq Data(10x Genomics; CG000234 Rev A). In this study, Cell type-specific cut site distribution was exported from Loupe Cell Browser (10x Genomics,) by loading the fragments.tsv.gz to peak viewer and exporting cut sites per cell type per window. Cell clusters were annotated using canonical markers of known cell types. Mapped the obtained cell cluster and the cellular marker gene that has been reported and included on the cell marker website(http://biocc.hrbmu.edu.cn/CellMarker/index.jsp).

\subsubsection{Peak Related Genes Annotation}

The detected peaks are open chromatin-rich regions with potential regulatory functions, and looking at the location of peaks and genes can provide insight into chromatin accessibility characteristics. We used bedtools(Quinlan and Hall, 2010) to map each peak to a gene level based on the closest transcription start site within 1000 bases upstream or 100 bases downstream of the TSS.

\subsubsection{Peak Related Genes GO Enrichment and Genes Pathway Enrichment Analysis}

We use Gene Ontology (GO) to analyze Peak-related Gene. GO has three ontologies: molecular function, cellular component, and biological process. The basic unit of GO is GO-term. Each GO-term belongs to a type of ontology. GO enrichment analysis provides all GO terms that significantly enriched in peak related genes compared to the genome background, and filter the peak related genes that correspond to biological functions. Firstly all peak related genes were mapped to GO terms in the Gene Ontology database (http://www.geneontology.org/), gene numbers were calculated for every term, significantly enriched $\mathrm{GO}$ terms in peak related genes comparing to the genome background were defined by hypergeometric test.GO terms meeting this condition were defined as significantly enriched GO terms in peak related genes. This analysis was able to recognize the main biological functions that peak related 
genes exercise. Genes usually interact with each other to play roles in certain biological functions. Pathway- based analysis helps to further understand genes' biological functions.

Here we use the Kyoto Encyclopedia of Genes and Genomes (KEGG) to made the Peak Related Genes Pathway Enrichment Analysis. KEGG is the major public pathway-related database. Pathway enrichment analysis identified significantly enriched metabolic pathways or signal transduction pathways in peak related genes compared with the whole genome background. The calculating formula is the same as that in the $\mathrm{GO}$ analysis.

\subsubsection{TF Motif Identification and Enrichment Analysis}

The peaks obtained are usually enriched in transcription factor (TF) binding sites, and the presence of certain groups can predict the activity of transcription factors. We use the MOODS(https://github.com/jhkorhonen/MOODS) The Python library packaged in Cell Ranger ATAC was used to scan each peak for matches to the group positions of transcription factors in the JASPAR database.

\section{Result}

\subsection{Quality control of the scATAC-seq profile}

We used the commercial system Chromium platform (10x Genomics) to performed scATAC-seq in nanoliter-sized droplets to measure single-cell chromatin accessibility landscape of Umbilical Cord Blood(Neal et al., 2018;Zheng et al., 2017b). Briefly, we disaggregated two cord blood mononuclear cells from the healthy control (NC) and the trisomy 18 syndrome (Edwards syndrome, ES) patient, and nuclei were isolated from each single-cell suspension and transposed in bulk with the transposase Tn5 (Figure 1.A). After removing additional cellular debris and minimizing mitochondrial contamination, a total of 11,611 cord blood mononuclear cells (6,315 cells of NC; 5,296 cells of ES) passing the filter yielded 5,689 median fragments, a $9.5 \%$ fraction of transposition events in peaks in the cell barcodes, and a $44.6 \%$ fraction of fragments overlapping the targeted region. The median number of unique fragments per cell barcode in the NC_CBMC library after normalization was 226,088,665, and that in the ES_CBMC library was $170,248,377$. More than 2-fold enrichment of fragments proximal to TSSs (relative to distal regions) from the NC_CBMC data and ES_CBMC data was observed. These data reflect a high fraction of fragments captured within open rather than closed chromatin (Figure 1.B and Figure 1.C). Since the Tn5 transposase preferentially attacks open chromatin regions, most DNAs were generally short fragments containing no or only one nucleosome, while the long fragments of NC_CBMC data and ES_CBMC data containing multiple nucleosomes exhibited a distinct fragment distribution in terms of content distribution. The library insertion size distribution for each sample was calculated using the comparison information from both ends of the reads (Figure 1.D and Figure 1.E). On average, at $27.8 \times 103$ unique fragments mapped to the nuclear genome, approximately $38.1 \%$ of Tn5 insertions were within peaks 
present in aggregated profiles from all cells, a proportion comparable to published acceptable ATAC-seq profiles.

\subsection{Single-cell chromatin accessibility profile of Umbilical Cord Blood from major immune cell types}

The sc-ATAC-seq reads from 11,611 cells from both NC and ES use Cell Ranger ATAC and UMAP cluster to perform dimensionality reduction clustering and identified a total of 13 cell clusters. Mapped the obtained cell cluster and the cellular marker gene that has been reported and included on the cell marker website (http://biocc.hrbmu.edu.cn/CellMarker/index.jsp), also, marker gene identification and cell specific-type annotation were performed according to the Cell Type Annotation Strategies for Single Cell ATAC-Seq Data(10x Genomics; CG000234 Rev A). We assigned the 13 cell clusters identified as the 6 major immune cell types known to be found in the blood: T cells, B cells, Monocytes/ Dendritic cell (DC), Natural Killer cells (NK), Progenitor cells, Megakaryocyte Erythroid(ME)(Figure 2A, Supplementary Figure S1A, and Supplementary Figure S1B). Analysis of total 13 cell clusters identified 6 distinct populations of cells which are demonstrated accessibility of open chromatin regions linked to cell-specific genes: $B$ cells were assigned by MS4A1, CD79A, and $C D 79 B$ gene promoter cell-specific genes(Yao et al., 2014). Cell populations expressing ITGAX, CD36, and IL3R1 were indefiend as monocytes/ dendritic cells (DC) (Cabillic et al., 2006) (Sinha et al., 2018). Natural killer(NK) cells were identified by GZMB and NKG7gene promoter cell-specific genes. T cells were identified by $L E F 1$ gene promoter cell-specific genes(Zheng et al., 2017a), and megakaryocyte Erythroid(ME) cells were identified by KIT, CD36,CD38(https://www.biorad-antibodies.com/human-immune-cell-markers-selection-tool.html\#cell=megakaryocyte-erythroid-cells) gene promoter cell-specific genes(Kellner et al., 2015;Sinha et al., 2018). Progenitor cells were identified by PECAM1 cell-specific genes(Cervelló et al., 2011)(Figure 2C). After determining the 6 cell types that belonged to the seven cell clusters of cord blood mononuclear cells, we evaluated cell abundances and calculated the composition ratio of each group of cells. Among the six major cell clusters, three were significantly larger in trisomy 18 syndrome. The ratio of B cells, progenitor cells, and megakaryocyte erythroid cells were significantly higher in the ES than the NC group. The ratios of natural killer (NK) cells, T cells, and monocyte/dendritic cell (ES) cells were significantly lower in the ES group than in the NC group (Figure 2D). In 1994, G. Makrydimas et al (Makrydimas et al., 1994) enumerated lymphocyte subpopulations in foetal blood obtained by cordocentesis from eight trisomy 18 fetuses at 20-36 weeks' gestation. Compared with values in chromosomally normal foetuses, in trisomy 18 the mean $T$ and natural killer (NK) cell counts were significantly lower, while the mean B cell count was not significantly different. Here, the number of B cells was significantly increased in the disease group in our single-cell analysis. These findings demonstrate that in trisomy 18 there is abnormal intrauterine development of the immune system. We next counted the cellular composition of the ES and NC groups (Figure 2E). Our results suggest that certain genes in B cells, progenitor cells, and megakaryocyte erythroid (ME) cells play an essential role in the development of the ES phenotype. Similarly, Anindita Roy's (Roy et al., 2012) showed that the 40-fold increase in childhood Megakaryocyte-Erythroid and Down syndrome implicates 
trisomy 21 (T21) in perturbing fetal hematopoiesis. To determine the differences in the number of differentially accessible peak regions between the six classes of identified cell populations, we performed volcano mapping analysis. Compared to controls (NC), a total of 494 differentially open regions were obtained in the ME cell populations of trisomy 18 (ES), of which 160 genes $(p<0.05, \log 2 F C>1)$ and 334 genes expression $(p<0.05, \log 2 \mathrm{FC}<-1)$ (Figure $2 \mathrm{~F})$. A total of 174 significantly different peaks were obtained from the progenitor cells, of which 43 indicated upregulation $(p<0.05, \log 2 F C>1)$ and 131 peaks downregulation $(p<0.05, \log 2 \mathrm{FC}<-1)$ (Figure $2 \mathrm{G}$ ). This result pointed us in the direction of our next analysis by implying that ME and progenitor cell populations play important roles in the development of trisomy 18 , followed by a further analysis of the heterogeneity of ME cell populations and P cells in the control and disease subjects.

\subsection{Human T-cell leukemia virus 1 infection pathway disorder in Megakaryocyte Erythroid cells}

We identified a total of 267 megakaryocyte erythroid (ME) cells. The proportion of megakaryocyte erythroid (ME) cells in ES and NC was $0.461(244 / 5296)$ and $0.035(22 / 6315)$, respectively. We found a significantly higher proportion of ME cells in the cord blood of patients with trisomy 18 than controls. This may be due to the presence of additional chromosomes in trisomy 18 and may be related to some of the phenotypes of trisomy 18 . The clinical significance of the significantly increased proportion of ME cells in the disease group is unclear. However, we note that Roy A etc.al(Roy et al., 2012) reported that a 40-fold increase in childhood megakaryocyte-erythroid and B-cell leukemia in Down syndrome implicates trisomy 21 (T21) in perturbing fetal hematopoiesis. The increased number of ME cell populations suggestive of the disease group may be related to the phenotype of trisomy 18 , such as tumors. We were motivated to dig deeper into the transcription factors in ME cell populations that would cause differences in gene expression. We were motivated to dig deeper into the transcription factors expressed in the ME cell population that would cause differences in gene expression. We performed a further UMAP (uniform manifold approximation and projection) analysis of the ME cell population(Becht et al., 2018). We annotated the genes regulated by the transcription factors of the differential peak regions, and the analysis of the genes co-regulated by the significantly differential peaks of ME- 0 and ME- 1 yielded 255 genes co-regulated by these differential peaks (Figure 3C). We counted the number of genes upregulated by ME- 0 and ME- 1 (Figure 3D). To obtain the number of regions of differentially accessible peaks for ME0 and ME-1, we performed a volcano diagram analysis, and we obtained a total of 472 significant different genes (89 upregulated, 383 downregulated, $\mathrm{p}<0.05,|\log 2 \mathrm{FC}|>1$ ) for $\mathrm{ME}-0$ and 938 significant different genes (270 upregulated, 668 downregulated, $p<0.05$, |log2FC| $>1$ ) for ME-1 (Figure 3E and Figure $3 F)$. To identify the differential functions and related pathways of the two populations of ME cells, we performed $G O$ and KEGG analysis of the neighbouring genes of the significantly different peaks ( $p$ adjust $<0.05,|\log 2 \mathrm{FC}|>1)$. The $\mathrm{GO}$ analysis showed that the differentially expressed genes of ME cells were associated with biological functions such as negative regulation of phosphorylation and negative regulation of protein phosphorylation (Figure 3G). Denise Utsch Gonçalves team(Gonçalves et al., 2010) 
reported that Human T-cell leukemia virus type 1 (HTLV-1) is the first human retrovirus to be discovered, it causes serious diseases in humans, including adult T-cell leukemia/lymphoma (ATL) and a disabling neurological disorder (HTLV-associated myelopathy/tropical spastic paraplegia [HAM/TSP]), in addition to other diseases such as retinitis, rheumatic syndrome, susceptibility to helminthic, and bacterial infections. Our analysis of the gene enrichment pathway regulated by the open regions of the peaks implies that an increased ME cell population in trisomy 18 patients causes aberrant regulation of the human T-cell leukaemia virus 1 infection signalling pathway. Human T-cell leukaemia virus 1 infection causes serious human diseases, such as leukaemia, which describes the tumour features of trisomy 18 syndrome(Satgé et al., 2016;Younes et al., 1994). Our results suggest that transcriptional dysregulation contributes to the development and progression of the trisomy 18 phenotype, that additional chromosomes may contribute to the increase in ME cell populations, and that genes of the human T-cell leukaemia virus 1 infection pathway are dysregulated, but the mechanism of transcriptional deregulation is unclear. Further clarification is needed

\subsection{Down-regulation of MCM3 and CCNB2 in Progenitor cells}

Cord blood contains a large number of stem cells, including haematopoietic stem cells and a variety of other stem cells, collectively known as cord blood stem cells/progenitor cells. We captured a total of 538 progenitor cells, including 500 in the ES group and 38 in the NC group (Figure 1B and C). After differential analysis, 1070 genes were obtained, among which 43peaks were significantly upregulated and 131 peaks were significantly downregulated $(p<0.05, \log 2 \mathrm{FC}>1)$, and there was no significant difference for 896 peaks (Figure 2G). To assess key genes for transcription factor-inaccessible regions of the P-cell population, we used the cytoHubba program of Cytoscape (3.8.0) to calculate the hub genes (top 10, $\mathrm{MCC} /$ degree) (Figure 4A) Among them, Cyclin B2 (CCNB2) is a member of the cyclin family and is an essential component of the cell cycle regulatory machinery. Cyclin B2 may play a key role in transforming growth factor beta-mediated cell cycle control(Lei et al., 2016). The downregulation of CCNB2 may lead to chromosome segregation abnormalities in trisomy 18. MCM3 (Minichromosome Maintenance Complex Component 3 ) is a highly conserved mini-chromosome maintenance protein (MCM) that is involved in the initiation of eukaryotic genome replication(Murai et al., 2018). The hexameric protein complex formed by MCM proteins is a key component of the prereplication complex and may be involved in the formation of replication forks and the recruitment of other DNA replication-related proteins. Diseases associated with MCM3 include grade III astrocytoma and lung cancer. Among its related pathways are the regulation of activated PAK-2p34 by proteasome-mediated degradation and the E2F transcription factor network. To further investigate the molecular characteristics of the trisomy 18 progenitor cells, we performed a UMAP (uniform manifold approximation and projection) analysis of the progenitor cells (Figure 4B). To obtain genes differentially expressed in trisomy 18, we performed volcano mapping analysis of three cell populations of $\mathrm{P}$ cells, and we obtained the $\mathrm{P}-\mathrm{O}$ and $\mathrm{P}-1$ differentially expressed genes $(P<0.05$, log2FC $>1)$, but no significant differences for $P-3$. Compared with the trisomy 18 
control, the trisomy 18 samples had 233 significantly different genes in $\mathrm{P}-0$, including 164 downregulated genes $(P<0.05, \log 2 F C<-1)$ and 69 upregulated genes $(P<0.05, \log 2 F C>1)$ (Figure $4 D)$, and 343 significantly different genes in $P-1$, including 27 downregulated genes $(P<0.05, \log 2 F C<-1)$ and 336 upregulated genes 316 genes $(P<0.05, \log 2 F C>1$ ) (Figure $4 E)$. We identified 15 motifs that were differentially expressed in both P-0 and P-1 (Figure 4F). To determine the main biological functions performed by P-0 and P-1 differentially expressed genes, we performed a GO enrichment analysis of the differentially expressed genes. The results showed that these differentially expressed genes primarily regulated the cell cycle phase transition, mitotic cell cycle phase transition, and nucleocytoplasmic transport. It has been shown (Paton et al., 1974), compared to normal diploid cells, G2 may be approximately 3 times longer in trisomy cells than in normal cells. Our results indicate that the genes that control the mitotic cycle in trisomy 18 have altered chromatin accessibility, and our analysis yielded key genes related to the cell cycle: CCNB2 and MCM3, which have been reported to be essential components of the cell cycle regulatory machinery, and the latter is one of the highly conserved mini-chromosome maintenance proteins

\section{Conclusion}

Here, we set out to generate a single-cell atlas of umbilical cord blood mononuclear cell chromatin accessibility. We applied sc-ATAC-seq to measure chromatin accessibility in 11,611 single cells derived from 2 samples, representing 6 major immune cell types. From the sc-ATAC-seq data, we identified diverse cell types, defined candidate cell-specific markers, and revealed the transcription factor (TF) regulatory network that specifies each cell type. Compared with those from the healthy donor (NC), a total of 494 (160 upregulated, 334 downregulated, $\mathrm{P}<0.05$, log2FC $>2$ ) genes in megakaryocyte erythroid cells were differentially expressed in the trisomy 18 syndrome (ES) donor, and a total of 174 (43 upregulated, 131 downregulated, $\mathrm{P}<0.05, \log 2 \mathrm{FC}>2)$ genes in progenitor cells were differentially expressed in the trisomy 18 syndrome (ES) donor. We also used these data to link distal regulatory elements to their target genes, characterize umbilical cord blood mononuclear cell heterogeneity in chromatin accessibility within cell types, and identify transcription factors (TFs) of principal relevance to trisomy 18 syndrome. We found that CCNB2 and MCM3 may be vital to the development of trisomy 18 . We identified a pathway in which the master differential regulatory pathway in the ME cell population involves human T-cell leukaemia virus 1 infection, a pathway that is dysregulated in patients with trisomy 18 and which we believe may lead to leukaemia in patients with trisomy 18 . The biological function of differentially expressed genes in P-cell populations, those with a higher degree of accessibility to chromatin regions, was associated with cell cycle regulation and chromatin stability, and we also identified two meaningful transcription factors, CCNB2 and MCM3, which have been reported to be essential components of the cell cycle and chromatin.

\section{Discussion}


To characterize the differences in chromatin accessibility and the biological functions and pathways implicated in the disease state of trisomy 18, we performed single-cell ATAC sequencing of cord blood band nuclei and performed reagent-free identification of the major cell types. Our study has at least two limitations. First, due to the low availability of cord blood, only one healthy donor and one trisomy 18 foetal donor were available, which is hardly representative of the true characteristics of trisomy 18 . At this stage, we can only characterize the common cell types. Our cell type assignments should be considered preliminary and ad hoc, and such assignments might vary considerably between independent studies. More detailed work (for example, further scaling up, protocol improvement, etc.) will be necessary to efficiently dissect chromatin accessibility in rare cell types(Cusanovich et al., 2018). Nonetheless, our study reveals important molecules that may be associated with the development of trisomy 18: CCNB2 and MCM3 may be vital. These findings provide candidate molecules for future studies of trisomy 18 . We

found some differences in genes involved in pathways that may cause disease. For example, in human Tcell leukaemia virus 1 infection, although the specific mechanism by which the changes occur is unclear, the results suggest that the regulation of this pathway in trisomy 18 is altered. We also obtained several hundred genes that are differentially expressed from inaccessible chromatin regions of ME and $\mathrm{P}$ cells. How these genes affect the occurrence and development of trisomy 18 is not yet known, but our findings will provide a molecular reference for future studies. It is worth further elucidating the specific role of these molecules in the development of trisomy 18 phenotypes.

\section{Author Contributions}

$\mathrm{XQ}$ interpreted the data and wrote the main manuscript text. $\mathrm{YH}, \mathrm{HW}$ and $\mathrm{ZH}$ contributed to the interpretation of the data. JZ and WC performed the experiments. WX and JC designed and conceived the experiments. MY and QY reviewed the manuscript. WD and HL performed the data analysis. YD and DT supervised the experiments and contributed to the interpretation of the data.

\section{Data Availability}

The raw and processed data in this manuscript have been deposited with the Gene Expression Omnibus under accession number GSE158178. Related website is: https://www.ncbi.nlm.nih.gov/geo/query/acc.cgi?acc=GSE158178.

\section{Funding}

This work was supported by the Science and Technology Planning Project of Guangdong Province, China 『No. 2017B020209001》, the science and technology plan of Shenzhen (No. JCYJ20180305163846927), Guangxi Key Laboratory of Metabolic Diseases Research (20-065-76).

\section{Acknowledgements}

We thank Li Chen from Hangzhou LC-BIO Co., Ltd. for technical assistance and analysis guidance. 


\section{Ethics Declarations \\ 10.1 Ethics approval and consent to participate}

The Institutional Review Board of the Shenzhen People's Hospital approved the current study, and the study protocol adhered to the Declaration of Helsinki. Written informed consent was given by all participants. Ethical review number of this study:LL-KT-2018356.

\subsection{Consent for publication}

Not applicable

\subsection{Competing interests}

The authors declare that they have no competing interests.

\section{References}

Becht E, Mclnnes L, Healy J, Dutertre CA, Kwok IWH, Ng LG, et al. (2018). Dimensionality reduction for visualizing single-cell data using UMAP. Nature biotechnology,

Buenrostro JD, Giresi PG, Zaba LC, Chang HY, Greenleaf WJ. (2013). Transposition of native chromatin for fast and sensitive epigenomic profiling of open chromatin, DNA-binding proteins and nucleosome position. Nat Methods, 10:1213-1218.

Cabillic F, Rougier N, Basset C, Lecouillard I, Quelvennec E, Toujas L, et al. (2006). Hepatic environment elicits monocyte differentiation into a dendritic cell subset directing Th2 response. Journal of hepatology,44:552-559.

Carey JCJMogs. (2005). Trisomy 18 and trisomy 13 syndromes.

Cereda A, Carey JC. (2012). The trisomy 18 syndrome. Orphanet J Rare Dis,7:81.

Cervelló I, Mirantes C, Santamaria X, Dolcet X, Matias-Guiu X, Simón C. (2011). Stem cells in human endometrium and endometrial carcinoma. International journal of gynecological pathology : official journal of the International Society of Gynecological Pathologists,30:317-327.

Clevenger J, Chavarro C, Pearl SA, Ozias-Akins P, Jackson SA. (2015). Single Nucleotide Polymorphism Identification in Polyploids: A Review, Example, and Recommendations. Mol Plant,8:831-846.

Criscuolo A, Brisse S. (2013). AlienTrimmer: a tool to quickly and accurately trim off multiple short contaminant sequences from high-throughput sequencing reads. Genomics,102:500-506. 
Cusanovich DA, Hill AJ, Aghamirzaie D, Daza RM, Pliner HA, Berletch JB, et al. (2018). A Single-Cell Atlas of In Vivo Mammalian Chromatin Accessibility. Cell,174:1309-1324.e1318.

Edwards JH, Harnden DG, Cameron AH, Crosse VM, Wolff OH. (1960). A new trisomic syndrome. Lancet, 1:787-790.

Fisher JM, Harvey JF, Morton NE, Jacobs PA. (1995). Trisomy 18: studies of the parent and cell division of origin and the effect of aberrant recombination on nondisjunction. Am J Hum Genet,56:669-675.

FitzPatrick DR, Ramsay J, McGill NI, Shade M, Carothers AD, Hastie ND. (2002). Transcriptome analysis of human autosomal trisomy. Human molecular genetics,11:3249-3256.

Goel N, Morris JK, Tucker D, de Walle HEK, Bakker MK, Kancherla V, et al. (2019). Trisomy 13 and 18Prevalence and mortality-A multi-registry population based analysis. Am J Med Genet A,179:2382-2392.

Gonçalves DU, Proietti FA, Ribas JG, Araújo MG, Pinheiro SR, Guedes AC, et al. (2010). Epidemiology, treatment, and prevention of human T-cell leukemia virus type 1-associated diseases. Clinical microbiology reviews,23:577-589.

González J, Muñoz A, Martos G. (2016). Asymmetric latent semantic indexing for gene expression experiments visualization. J Bioinform Comput Biol,14:1650023.

Kellner J, Li S, Zweidler-McKay PA, Shpall EJ, McNiece I. (2015). Phenotypic and functional comparison of mobilized peripheral blood versus umbilical cord blood megakaryocyte populations. Cytotherapy, 17:418-427.

Kobak D, Berens P. (2019). The art of using t-SNE for single-cell transcriptomics. Nature communications, $10: 5416$.

Kupke KG, Müller U. (1989). Parental origin of the extra chromosome in trisomy 18. Am J Hum Genet,45:599-605.

Lei CY, Wang W, Zhu YT, Fang WY, Tan WL. (2016). The decrease of cyclin B2 expression inhibits invasion and metastasis of bladder cancer. Urologic oncology,34:237.e231-210.

Makrydimas G, Plachouras N, Thilaganathan B, Nicolaides KH. (1994). Abnormal immunological development in fetuses with trisomy 18. Prenat Diagn,14:239-241.

Murai J, Tang SW, Leo E, Baechler SA, Redon CE, Zhang H, et al. (2018). SLFN11 Blocks Stressed Replication Forks Independently of ATR. Molecular cell,69:371-384.e376.

Neal JT, Li X, Zhu J, Giangarra V, Grzeskowiak CL, Ju J, et al. (2018). Organoid Modeling of the Tumor Immune Microenvironment. Cell,175:1972-1988.e1916. 
Paton GR, Silver MF, Allison AC. (1974). Comparison of cell cycle time in normal and trisomic cells. Humangenetik,23:173-182.

Quinlan AR, Hall IM. (2010). BEDTools: a flexible suite of utilities for comparing genomic features. Bioinformatics,26:841-842.

R Z, L H, L W, M C, W L, R L, et al. (2013). Gene expression analysis of induced pluripotent stem cells from aneuploid chromosomal syndromes.S8.

Roy A, Cowan G, Mead AJ, Filippi S, Bohn G, Chaidos A, et al. (2012). Perturbation of fetal liver hematopoietic stem and progenitor cell development by trisomy 21. Proceedings of the National Academy of Sciences of the United States of America,109:17579-17584.

Satgé D, Nishi M, Sirvent N, Vekemans M. (2016). A tumor profile in Edwards syndrome (trisomy 18). Am J Med Genet C Semin Med Genet,172:296-306.

Satpathy AT, Granja JM, Yost KE, Qi Y, Meschi F, McDermott GP, et al. (2019). Massively parallel single-cell chromatin landscapes of human immune cell development and intratumoral T cell exhaustion. Nature biotechnology,37:925-936.

Schep AN, Buenrostro JD, Denny SK, Schwartz K, Sherlock G, Greenleaf WJ. (2015). Structured nucleosome fingerprints enable high-resolution mapping of chromatin architecture within regulatory regions. Genome research,25:1757-1770.

Sinha D, Kumar A, Kumar H, Bandyopadhyay S, Sengupta D. (2018). dropClust: efficient clustering of ultra-large scRNA-seq data. Nucleic Acids Res,46:e36.

Smith DW, Patau K, Therman E, Inhorn SL. (1960). A new autosomal trisomy syndrome: multiple congenital anomalies caused by an extra chromosome. J Pediatr,57:338-345.

Stuart T, Butler A, Hoffman P, Hafemeister C, Papalexi E, Mauck WM, 3rd, et al. (2019). Comprehensive Integration of Single-Cell Data. Cell,177:1888-1902.e1821.

Torres EM, Williams BR, Amon A. (2008). Aneuploidy: cells losing their balance. Genetics,179:737-746.

Yao X, Williamson C, Adalsteinsson VA, D'Agostino RS, Fitton T, Smaroff GG, et al. (2014). Tumor cells are dislodged into the pulmonary vein during lobectomy. The Journal of thoracic and cardiovascular surgery, 148:3224-3231.e3221-3225.

Younes A, Jendiroba D, Engel H, Escudier S, Katz R, Rodriguez MA, et al. (1994). High incidence of monosomy 18 in lymphoid malignancies that have bone marrow and peripheral blood involvement. Cancer genetics and cytogenetics,77:39-44. 
Zhang R, Hao L, Wang L, Chen M, Li W, Li R, et al. (2013). Gene expression analysis of induced pluripotent stem cells from aneuploid chromosomal syndromes. BMC genomics,14 Suppl 5:S8.

Zheng C, Zheng L, Yoo JK, Guo H, Zhang Y, Guo X, et al. (2017a). Landscape of Infiltrating T Cells in Liver Cancer Revealed by Single-Cell Sequencing. Cell,169:1342-1356.e1316.

Zheng GX, Terry JM, Belgrader P, Ryvkin P, Bent ZW, Wilson R, et al. (2017b). Massively parallel digital transcriptional profiling of single cells. Nature communications,8:14049.

\section{Figures}


A

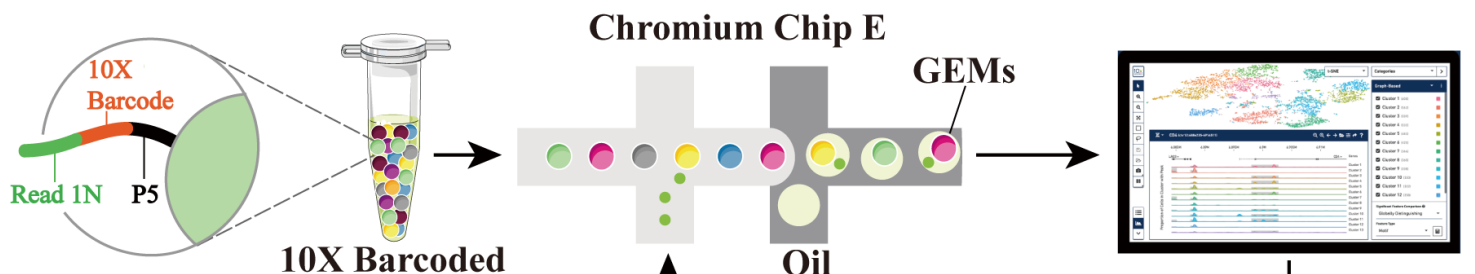

Gel Beads

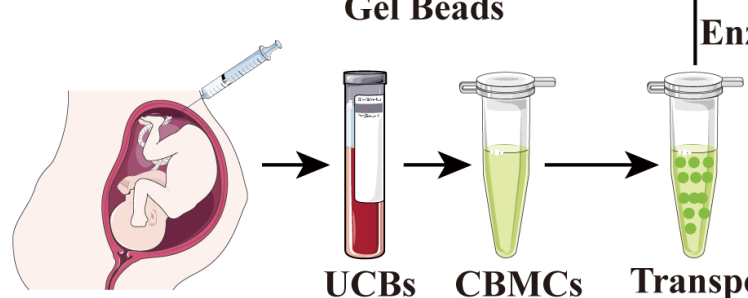
of Nuclei in Bulk

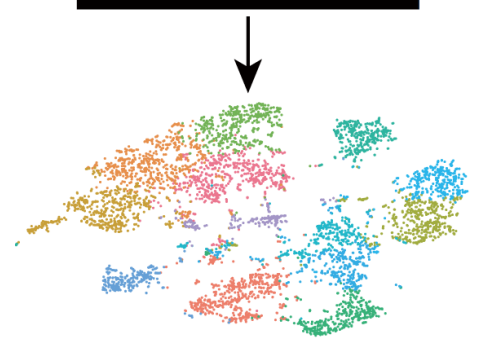

Clustering

B

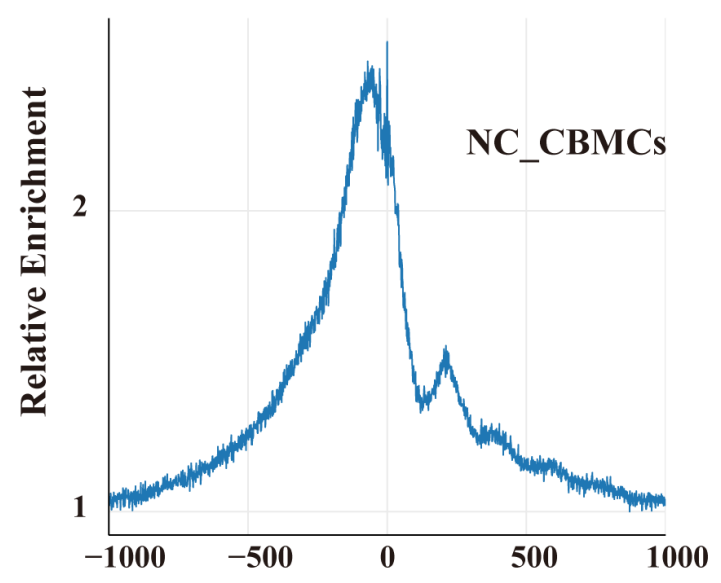

Enrichment around TSS(normalized)

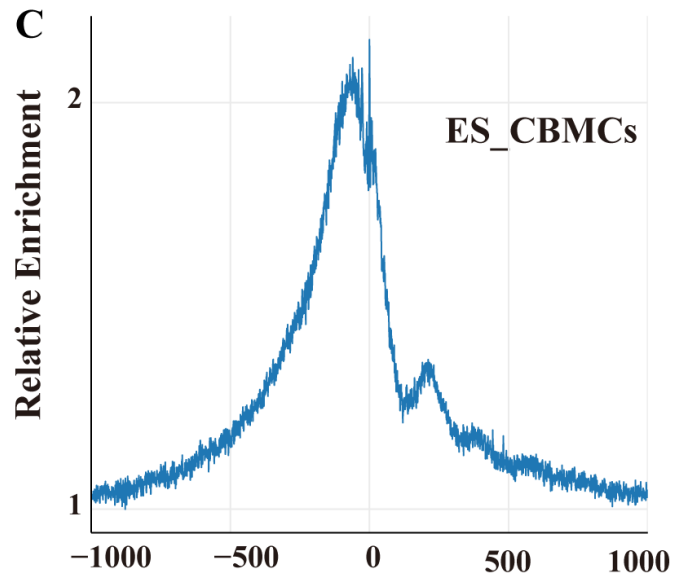

Enrichment around TSS(normalized)
D

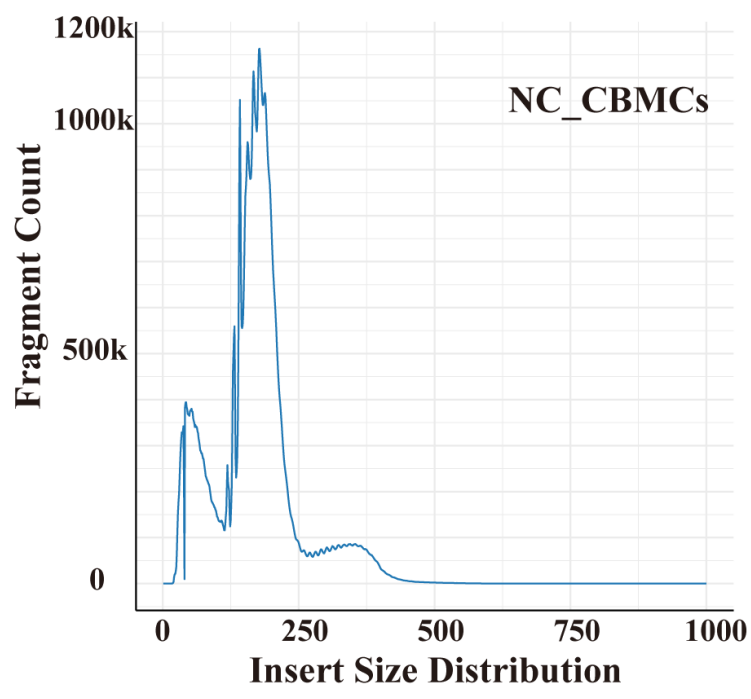

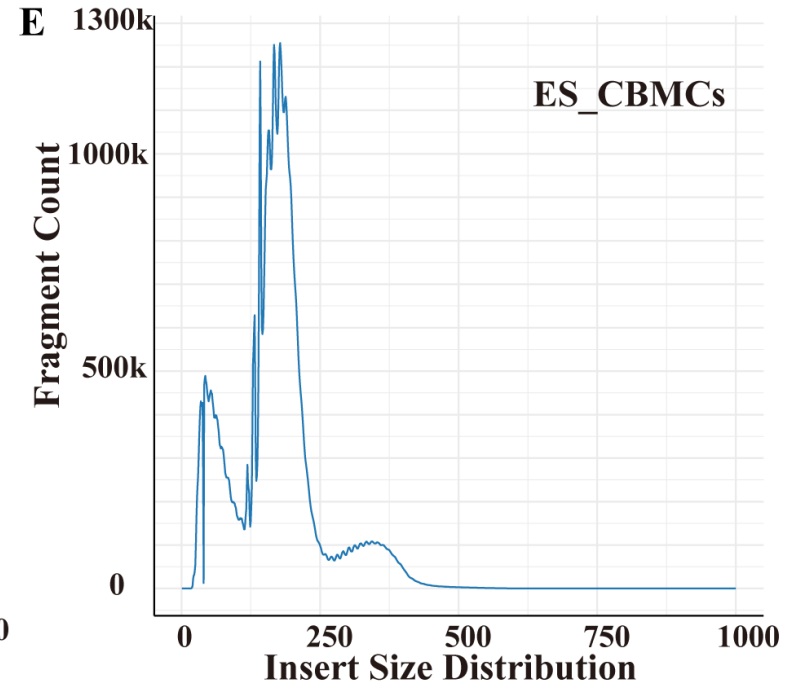

\section{Figure 1}

Quality control of the scATAC-seq profile. (A) Schematic of the single-cell assay for transposaseaccessible chromatin using sequencing of UBMC nuclei isolated and transposition. (B and $C$ ) The signal distribution map around TTS after normalization of NC_UBMCs and ES_UBMCs. The horizontal coordinate is the position relative to the TSS, and the vertical coordinate is the relative signal strength. (D and E) Length distribution of NC_UBMCs and ES_UBMCs library inserts for each sample. The horizontal 
axis indicates the length of the inserts, and the vertical axis indicates the proportion of inserts of that length to the total number of inserts.
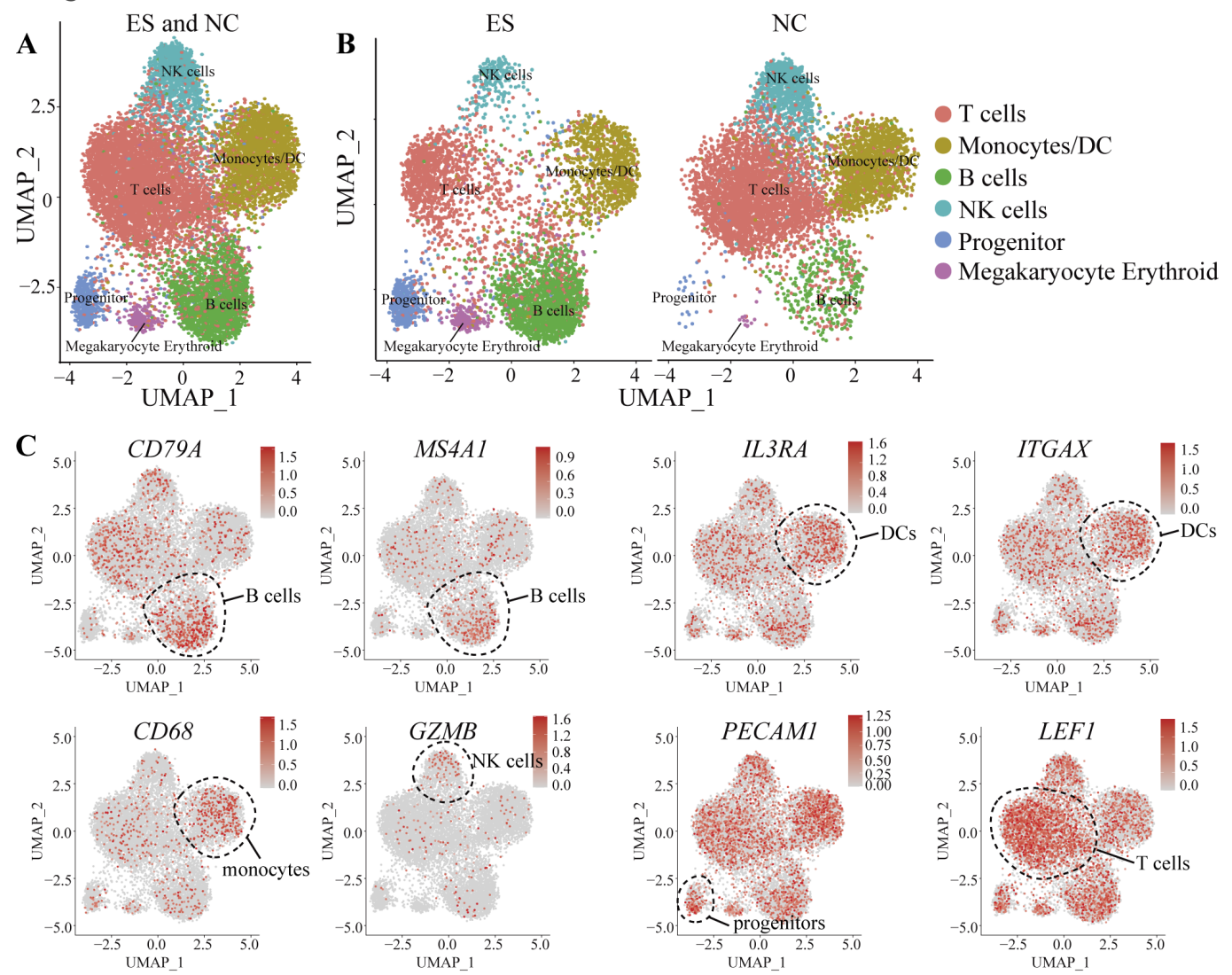

D
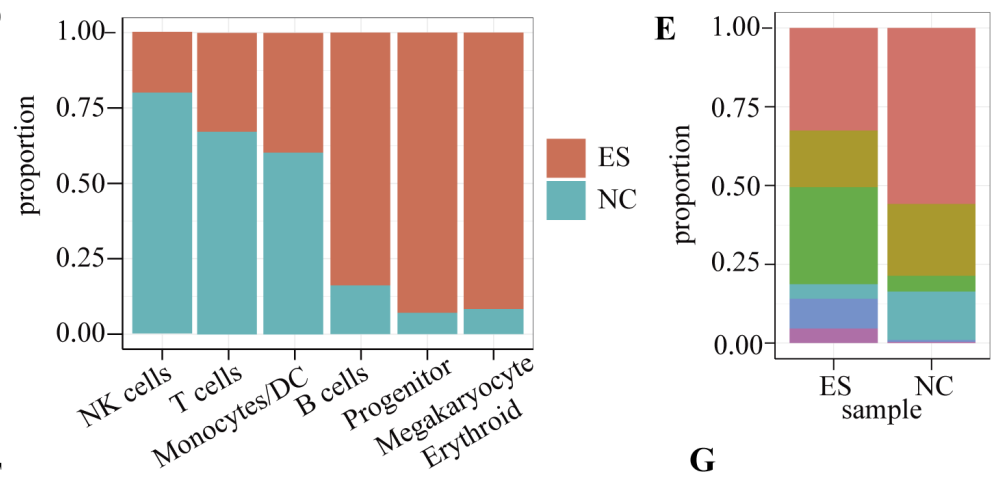

T cells

Monocytes/DC

B cells

Progenitor

Megakaryocyte Erythroid

F

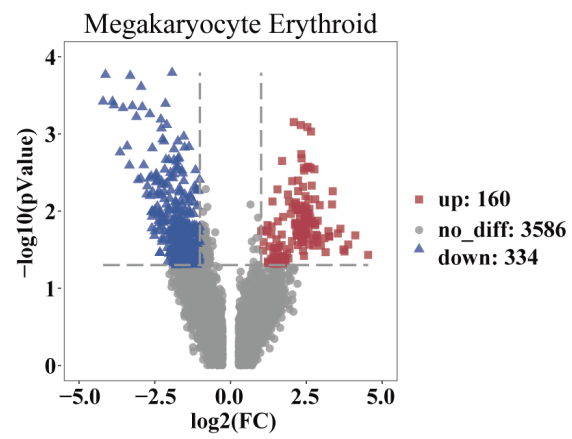

G

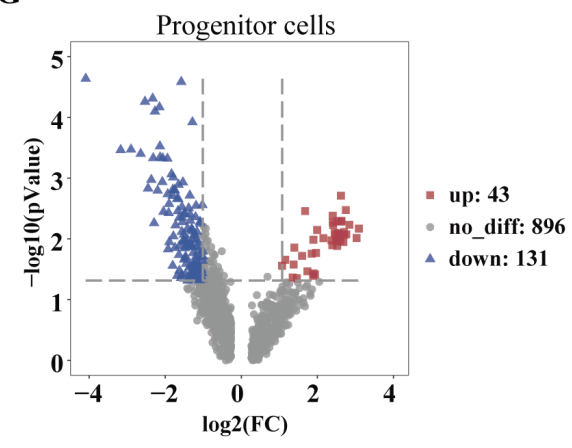

Figure 2

Landscape of trisomy 18 and control umbilical cord blood single nucleated cells. (A) UMAP of ES_Library and NC_Library. (B) UMAP of ES_Library and UMAP of NC_Library. (C) Cell-specific marker expression of 
clusters. (D) Cell ratio of ES_Library and NC_Library in sample. (E) Cell ratio of ES_Library and NC_Library in cell type. (F) Volcano plot of megakaryocyte erythroid and progenitor cells.

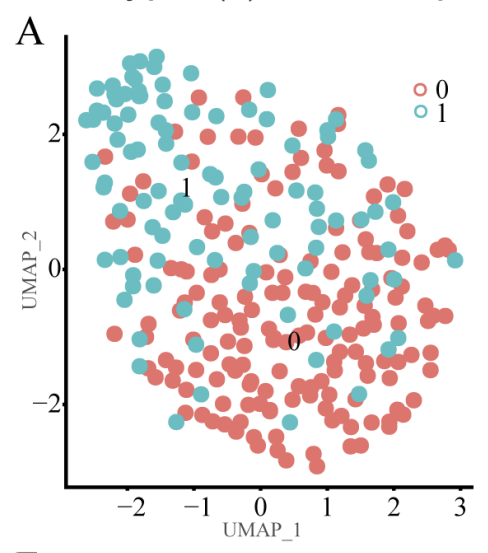

$\mathrm{E}$

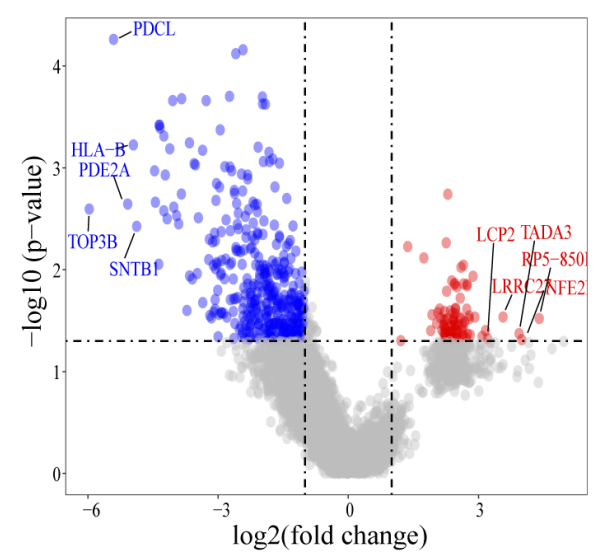

$\mathrm{C}$

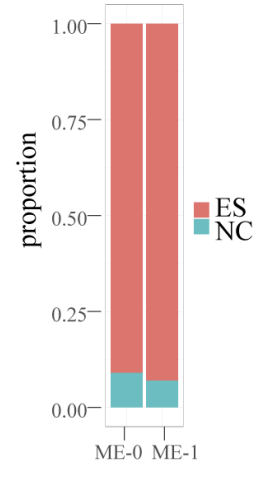

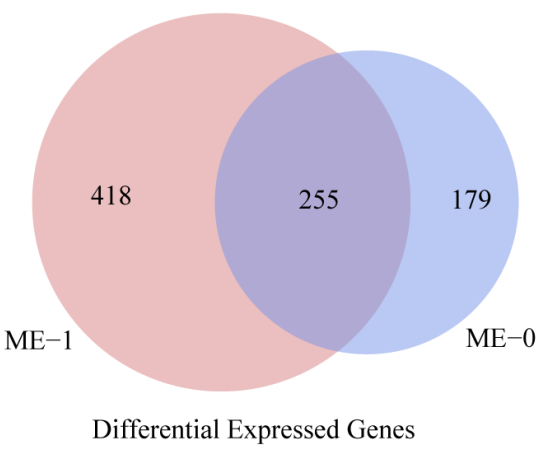

$\mathrm{D}$

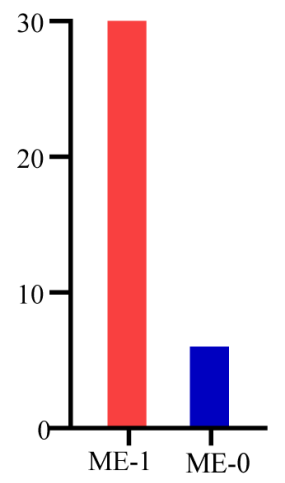

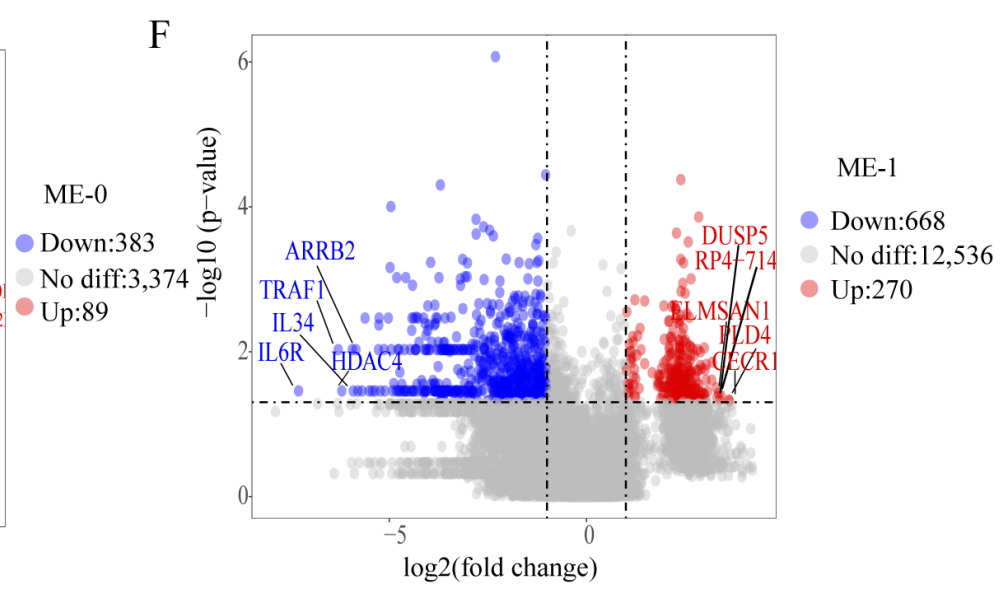

G

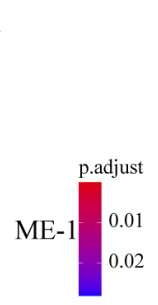

$$
\text { ME-0 } \overbrace{0.025}^{\substack{0.0005 \\ 0.005}}
$$

PML body mical synapse

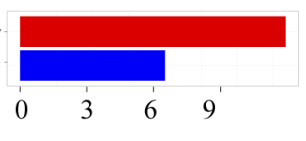

$\mathrm{H}$

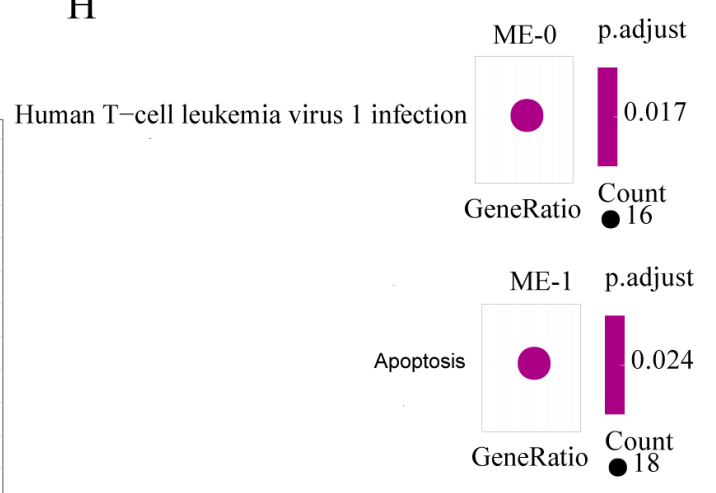
negative regulation of protein phosphorylation positive regulation of leukocyte differentiation positive regulation of hemopoiesis negative regulation of kinase activity negative regulation of transferase activity regulation of leukocyte differentiation negative regulation of protein kinase activity positive regulation of cell adhesion alpha-beta $\mathrm{T}$ cell activation involved in immune response alpha-beta $\mathrm{T}$ cell differentiation involved in immune response regulation of T-helper 2 cell differentiation response to peptide hormone histone deacetylation regulation of $\mathrm{T}$-helper cell differentiation $\mathrm{T}$ cell differentiation involved in immune response regulation of CD4-positive, alpha-beta $\mathrm{T}$ cell differentiation negative regulation of cold-induced thermogenesis $\mathrm{T}$-helper cell differentiation

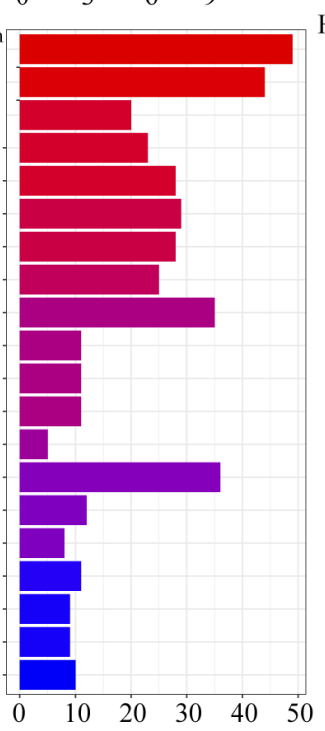
- 18

\section{Figure 3}

ME cell population clustering analysis with differentially accessible peak analysis. (A). UMAP plot of megakaryocyte erythroid cells. Cluster 0 is marked 0 , and cluster 1 is marked 1. (B). Proportions of cells in the ES and NC groups: megakaryocyte erythroid cells, cluster 0 labelled ME-0, cluster 1 labelled ME-1. (C). 
Genes coregulated by ME- 0 and ME- 1 had significantly differential peaks $(p<0.05, \log 2 F C>1)$. (D) Number of motifs significantly upregulated by ME- 0 and ME- $1(p<0.05, \log 2 F C>0.58)$. (E) ME-0 differentially expressed genes $(p<0.05,|\log 2 \mathrm{FC}|>1)$. Blue dots indicate downregulated genes, red dots indicate upregulated genes, and grey dots indicate genes that are satisfactorily significantly different and label the five genes with the largest multiplicity of differences. $(F)$ ME- 1 differentially expressed genes $(p<0.05$, $|\log 2 \mathrm{FC}|<1$ ). (G) Results of $\mathrm{GO}$ enrichment analysis of ME-0 and ME-1. (F) Results of KEGG enrichment analysis of ME-0 and ME-1.

A

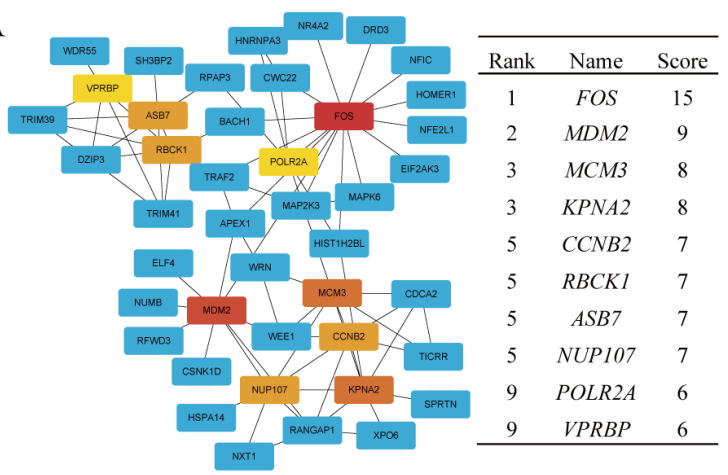

Progenitor cells Top 10 in network ranked by Degree method
B

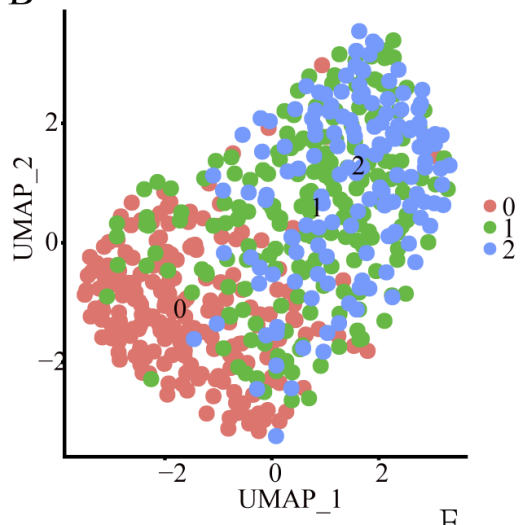

C

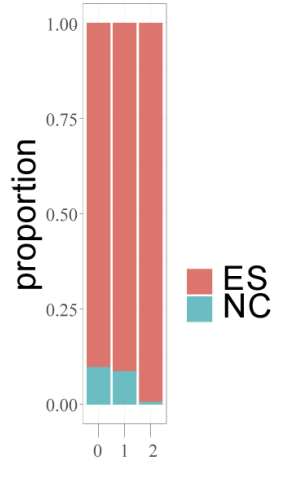

D

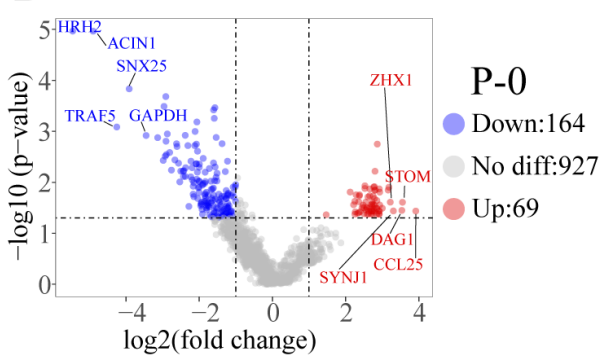

E

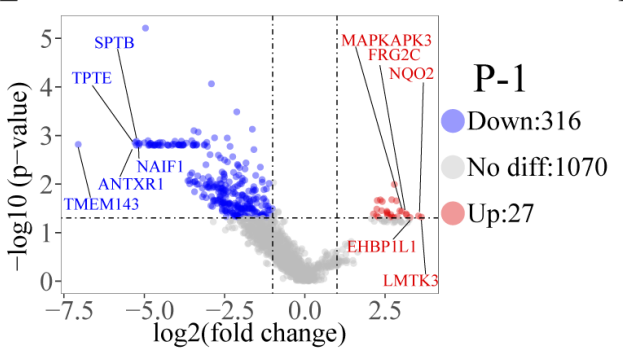

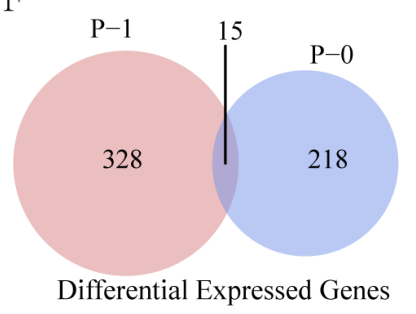

G

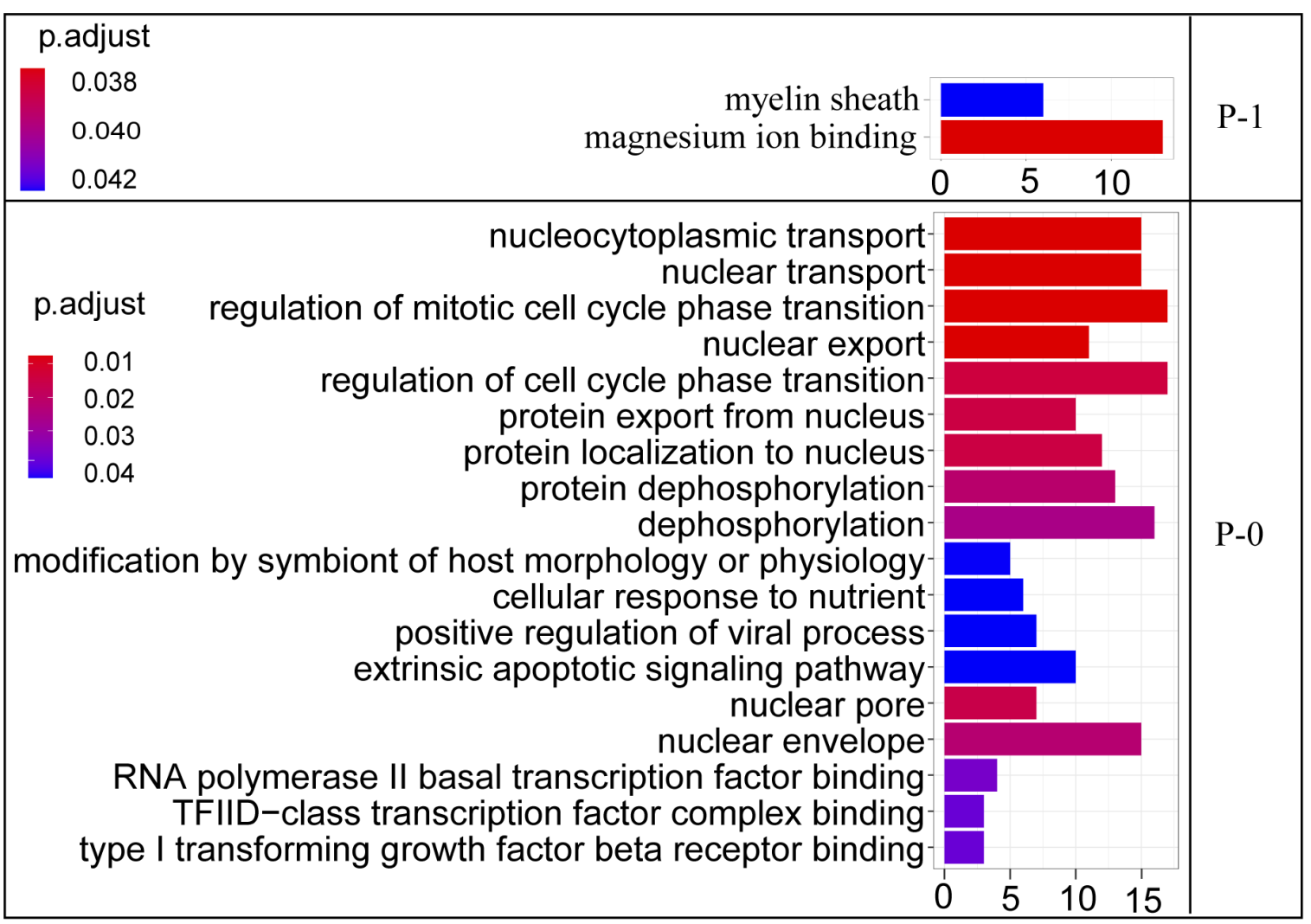




\section{Figure 4}

Molecular characterization of progenitor cells. (A) Top 10 network progenitor cells ranked by the degree method. The network diagram shows how the hub gene interacts with other genes. The redder the box, the higher the score, indicating a more critical gene. (B) UMAP plot of progenitor cells. Cluster 0 is marked 0 , and cluster 1 is marked 1 (C) Proportion of cells in the ES and NC groups in progenitor cells, cluster 0 labelled P-0, cluster 1 labelled P-1. (D) P-0 differentially expressed genes $(p<0.05,|\log 2 F C|>1)$. Blue dots indicate downregulated genes, red dots indicate upregulated genes, and grey dots indicate genes that are satisfactorily significantly different and label the five genes with the largest multiplicity of differences. $(E)$ P-1 differentially expressed genes $(p<0.05,|\log 2 F C|<1)$. ( $F)$ Genes co-regulated by P-0 and P-1 had significantly different peaks $(p<0.05, \log 2 F C>1)$. (G) Results of $G 0$ enrichment analysis of $P-0$ and $P-1$.

\section{Supplementary Files}

This is a list of supplementary files associated with this preprint. Click to download.

- SupplementaryMaterial.doc 